\title{
Gestion pastorale et structure des terroirs agricoles dans la périphérie de la Djona (Nord-Est Bénin)
}

\author{
Thierry Dèhouégnon HOUEHANOU ${ }^{1 *}$, Marcel HOUINATO ${ }^{1}$, Claude ADANDEDJAN ${ }^{1}$, \\ Armand Bienvenu GBANGBOCHE ${ }^{1}$, M. Sylvie HOUNZANGBE-ADOTE ${ }^{1}$ et \\ Brice Augustin SINSIN ${ }^{2}$ \\ ${ }^{1}$ Département de Production Animale (DPA), Faculté des Sciences Agronomiques (FSA), Université \\ d'Abomey-Calavi (UAC), Bénin. \\ ${ }^{2}$ Laboratoire d'Ecologie Appliquée (LEA), Faculté des Sciences Agronomiques (FSA), Université d'Abomey- \\ Calavi (UAC), Bénin. \\ *Auteur correspondant, E-mail: houehanout@yahoo.fr, Tel :022997213922
}

\section{RESUME}

En Afrique au Sud du Sahara, les systèmes d'élevage sont diversifiés et par conséquent leur étude s'avère indispensable. Ainsi, une enquête portant sur le système d'élevage bovin et l'étude de la structure des terroirs agricoles a été conduite dans l'arrondissement d'Angaradébou (zone périphérique de la Djona) du département de l'Alibori. L'étude a mis en exergue la dominance du sédentarisme comme mode de conduite, pratiqué dans un système agro-pastoral. L'effectif moyen a été de $46 \pm 20$ bovins par troupeau comprenant en majorité les zébus Keteeji et Tchiwali. Les ligneux fourragers recensés et épargnés dans les terroirs des éleveurs sont: Khaya Senegalensis, Afzelia africana, Pterocarpus erinaceus, Daniellia oliveri, Bombax costatum, Cordia sinensis et Acacia sieberiana et sont ceux intervenant dans l'alimentation des bovins dans la zone d'étude contrairement à Vitellaria paradoxa et à Parkia Biglobosa qui sont les plus épargnées dans les terroirs agricoles. Des terroirs agricoles répertoriés, les champs des éleveurs ont présenté le type le plus diversifié avec respectivement 2,02 bits et 0,72 pour l'indice de diversité de Shannon et l'équitabilité de Pielou. (C) 2008 International Formulae Group. All rights reserved.

Mots clés : Ligneux épargnés, mode de conduite, bovins, parcs, Bénin.

\section{INTRODUCTION}

L'élevage occupe une place de choix dans l'économie des pays d'Afrique subsaharienne par sa contribution d'environ 10 à $20 \%$ au PIB et 50 à $80 \%$ du PIB agricole (Faye et Alary, 2001). Au Bénin, sa participation au PIB est estimée à 6,2\% (MDR, 1994). Les systèmes d'élevage y sont très variés. On rencontre à la fois l'élevage extensif et amélioré, basé essentiellement sur l'utilisation de la végétation naturelle très peu entretenue, et ne permettant pas aux animaux d'exprimer leur potentiel génétique (Chaibou, 2005). Ainsi, la faible valeur alimentaire des pâturages tropicaux constitue un handicap majeur et, ajoutée aux faibles performances génétiques des animaux, ne permettent pas d'obtenir chez les ruminants une bonne production de lait et de viande. Aussi les formes d'utilisation de l'espace autour des aires protégées sont-elles généralement concurrentes avec les objectifs de protection. Il s'avère nécessaire de comprendre les systèmes d'élevage existant dans cette zone périphérique de la Djona afin d'une amélioration efficace.

Par ailleurs, les ressources alimentaires disponibles pour le bétail sont encore très peu connues dans les pays du Sud du Sahara et les ligneux fourragers offrent des perspectives très intéressantes du fait qu'ils constituent une composante importante de l'alimentation des 
bovins en saison sèche, notamment des espèces telles que Afzelia africana, Khaya senegalensis et Pterocarpus erinaceus (Houinato, 2001 ; Brisso et al., 2007). En Afrique, plusieurs études ont mis l'accent sur la variabilité des ressources et surtout le rôle des espèces ligneuses dans les bilans fourragers des systèmes d'élevage (Le Houerou, 1980 ; Piot, 1980 ; Sacko, 1991). Le rôle alimentaire et sanitaire de certains ligneux conservés ou non (Sinsin, 1991; Adandédjan, 1999; Houinato, 2001; Sieglstetter et Wittig, 2001) a également été par ailleurs mis en exergue en Afrique Subsaharienne humide.

$\mathrm{Au}$ Bénin, peu de littératures sont disponibles sur les systèmes d'élevage autour des aires protégées et les ligneux fourragers sont peu connus. L'objectif de cette étude est de caractériser l'élevage à la zone périphérique de la zone cynégétique de la Djona de même que la structure des terroirs agricoles riverains pour une gestion pastorale.

\section{MATERIEL ET METHODES \\ Milieu d'étude}

L'étude a été menée dans trois villages (Angaradébou, Thya et Alfakoara) situés à la périphérie de la Zone Cynégétique de la Djona (l'une des zones cynégétiques de la Réserve de Biosphère Transfrontalière du W) dans la commune de Kandi (département de l'Alibori), au Nord-Est du Bénin (figure 1 et 2). Cette zone s'étend entre $11^{\circ} 20^{\prime}$ et $11^{\circ} 50^{\prime}$ de longitude Ouest puis entre $2^{\circ} 20^{\prime}$ et $2^{\circ}$ 50 'de latitude Nord. On y rencontre plusieurs formations végétales parmi lesquelles: les forêts claires à Isoberlinia doka et différents types de savanes. Le climat est tropical sec de type soudano-sahélien ou Nord-soudanien caractérisé par une saison pluvieuse (6 mois) et une saison sèche. Les températures présentent une grande variation au cours de l'année. Le mois d'avril est le mois le plus chaud avec des températures moyennes minimales de $25^{\circ} \mathrm{C}$ à $28^{\circ} \mathrm{C}$ et maximales de $37^{\circ} \mathrm{C}$ à $41^{\circ} \mathrm{C}$ pour le même mois.

$\mathrm{La}$ population d'Angaradébou se compose de divers groupes ethniques. Adjakpa (1989) a identifié trois groupes: les Mokolés et les Dendis (en majorité sont principalement des agriculteurs), les Peuls (nombreux sont associés à toutes les ethnies et pratiquent l'élevage comme activité principale) et les Bariba, Haoussa, Yorouba et Fons (en minorité, sont principalement des artisans, commerçants et fonctionnaires). Selon le Centre Communal pour la Promotion Agricole (CeCPA) de Kandi, les principales cultures sont: le coton, le maïs, l'arachide et le sorgho représentant respectivement $50 \%$; $25,35 \% ; 11,90 \%$ et $12,82 \%$ des terres emblavées pour les cinq dernières campagnes agricoles. Quant à l'élevage bovin, 89167 têtes de bovin ont été contrôlées en 2006 par le Projet d'Appui au Développement de l'Elevage dans le Borgou (PADEB) dans la commune de Kandi dont 17,2\% dans l'arrondissement d'Angaradébou.

\section{Méthode}

Ndzingu Awa et al. (2004), a identifié dans les savanes d'Afrique Centrale six systèmes d'élevage sur la base de l'importance relative de l'agriculture et de l'élevage, des espèces prédominantes et des pratiques d'élevage. Ainsi la méthodologie adoptée a consisté en une enquête sur la description du système d'élevage bovin (mode de conduite, structure des troupeaux et gestion de l'espace), et les ligneux conservés. L'enquête a été semi informelle et a porté sur un échantillon de quinze (15) éleveurs Peuls (tous agro pastoraux) par village soit au total 45 enquêtés.

Un inventaire des peuplements ligneux a été réalisé et le dbh (diameter at breast height) des ligneux épargnés, mesuré dans quatre (4) placeaux de $50 \mathrm{~m}$ x $50 \mathrm{~m}$ installés au hasard par type de terroir. Au cours de l'inventaire toutes les espèces de ligneux émondées ont été recensées dans ces terroirs. Quatre types de terroirs agricoles soit 16 placeaux ont été évalués: les champs des agriculteurs (les Monkolés et les Dendis), les champs des éleveurs (les Peuls), les jeunes jachères et les vieilles jachères. Les terroirs agricoles ont été catégorisés ainsi en tenant compte de leur physionomie générale, des activités humaines et de l'importance des ligneux épargnés par les groupes socioprofessionnels. Les dbh supérieurs ou égales à dix $(\mathrm{dbh} \geq 10 \mathrm{~cm})$ ont été inventoriés. Les $\mathrm{dbh}<10 \mathrm{~cm}$ (régénération) n'ont pas été pris en compte dans ce travail. 


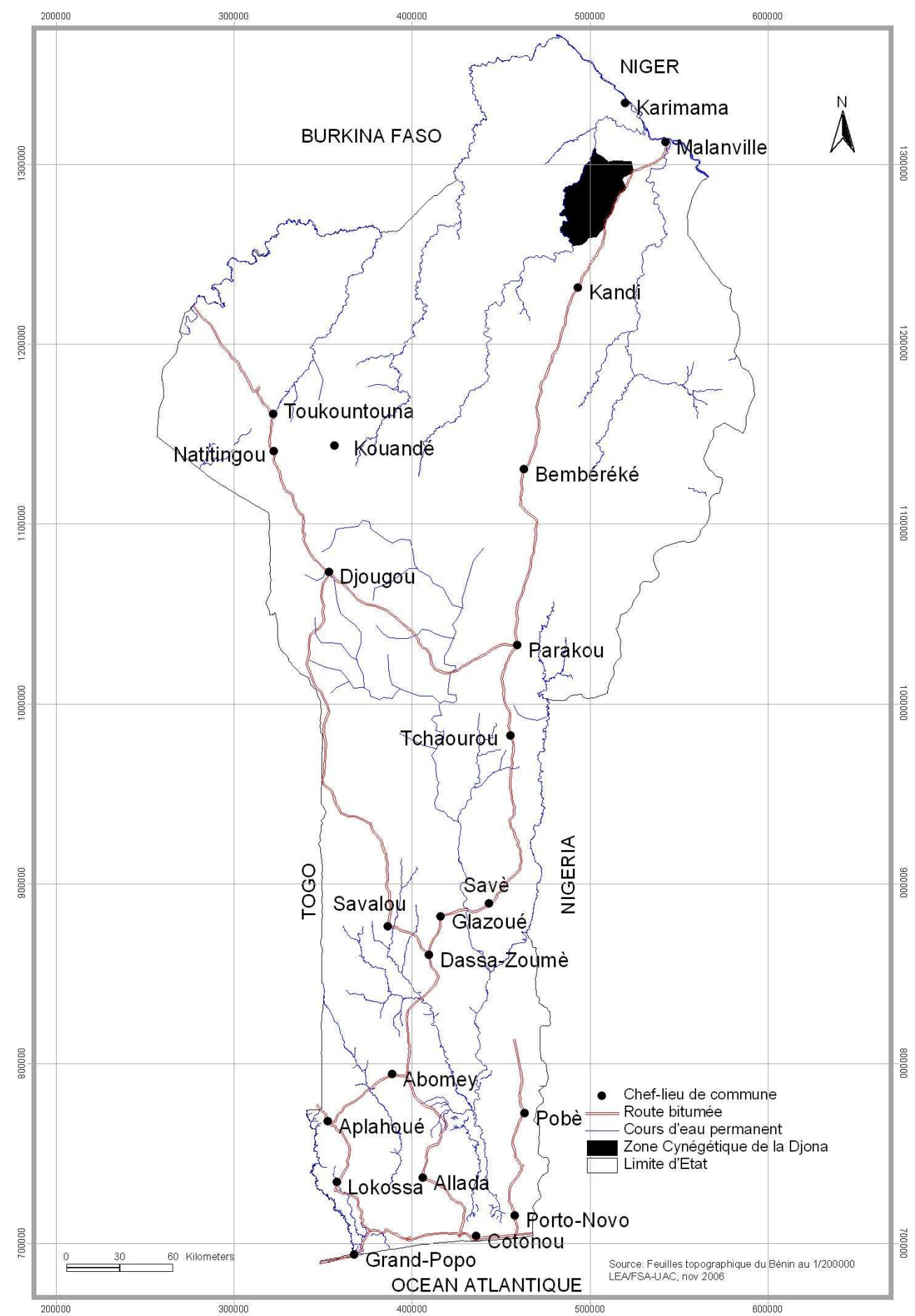

Figure 1: Carte de localisation de la zone Cynégétique de la Djona au Bénin. 


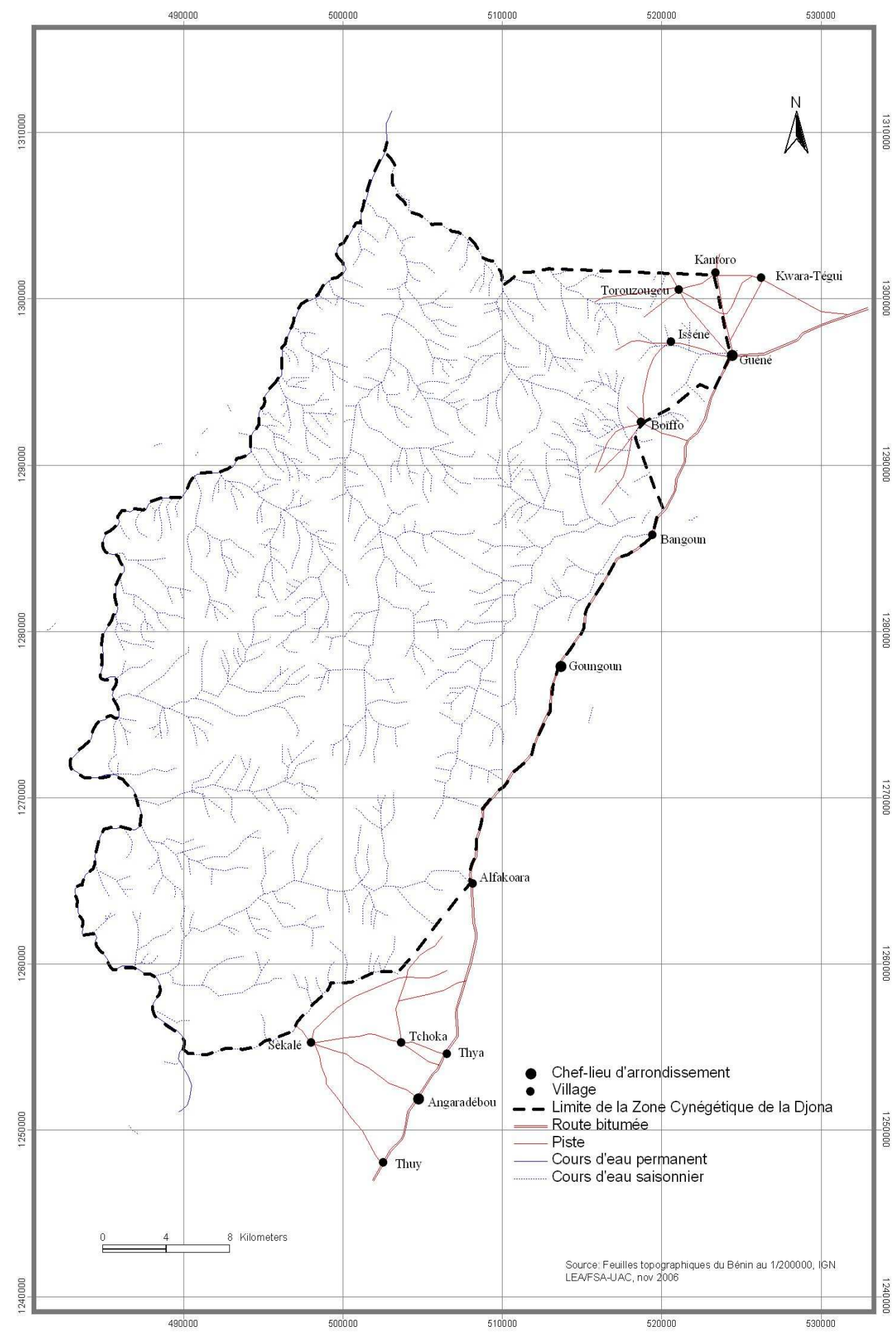

Figure 1: Carte de la zone cynégétique de la Djona et ses villages riverains. 
Les paramètres tels que la richesse spécifique (nombre d'espèce), la densité des ligneux (pieds/ha), les proportions des ligneux par espèce $(\%)$, et les indices de diversité de Shannon et de l'équitabilité de Pielou ont été évalués.

L'indice de diversité de Shannon Weiner $\left(\mathrm{H}=-\sum \pi \log _{2} \pi\right), \quad \pi$ représente la proportion des individus dans l'échantillon total qui appartiennent à l'espèce i. Cet indice est basé sur la théorie de l'information mutuelle. Exprimées en bit, les valeurs extrêmes sont comprises entre 0,5 et 4,5 bits environ.

L'indice d'équitabilité de Pielou $\left(E=\frac{H}{\log _{2} S}\right)$,

$S$ représente le nombre total des espèces dans le parc et $\mathrm{H}=$ indice de diversité de Shannon. La régularité correspond au rapport entre la diversité obtenue et la diversité maximale possible $(\log 2 \mathrm{~S})$ du nombre d'espèce $\mathrm{S}$. Elle varie entre 0 et 1 .

Le logiciel Excel 2003 a été utilisé pour calculer les moyennes, les écarts types, les indices de diversité et pour générer les graphiques d'illustration.

\section{RESULTATS}

Les résultats sont relatifs à (1) la gestion de l'espace, le mode de conduite et la structure des troupeaux et (2) la structure démographique des ligneux épargnés.

\section{Gestion de l'espace, mode de conduite et structure des troupeaux}

Les deux grandes zones exploitées par les éleveurs et les agriculteurs sont représentées par la zone tampon (zone à usage contrôlé) et la zone libre. La zone libre où les agriculteurs sont les propriétaires terriens, est plus sujette à la pression agricole que pastorale. $89 \%$ des éleveurs enquêtés pratiquent le sédentarisme comme mode d'élevage et détiennent en moyenne 46 bovins/troupeau avec un coefficient de variation de $43,47 \%$ alors que la transhumance (petite et grande) est le mode de conduite du reste des éleveurs $(11 \%)$ investis. La structure du cheptel est composée de vaches $(60 \%)$, de veaux (21\%), de taurillons et génisses $(13 \%)$, et de taureaux (6\%). Deux races traditionnelles de zébus (Bos taurus) sont rencontrées. Il s'agit des races Keteeji et "Tchiwali". La race "Tchiwali", la plus fréquente, a représenté plus de $60 \%$ de l'échantillon des éleveurs enquêtés.

\section{Structure démographique des ligneux épargnés \\ Le tableau 1 montre la structure} démographique des ligneux épargnés sur les terroirs agricoles. Les espèces listées dans ce tableau sont celles présentes dans les placeaux inventoriés. Toutes les espèces recensées qui sont épargnées ne sont pas systématiquement retrouvées dans les placeaux inventoriés. C'est le cas de Khaya senegalensis, Cordia sinensis et Acacia siberiana. Les espèces telles que Vitellaria paradoxa et Parkia biglobosa sont les plus épargnées dans les terroirs agricoles mais, le karité (Vitellaria paradoxa) a une forte représentation dans tous les terroirs agricoles inventoriés. Par ailleurs les espèces telles que Afzelia africana, Bombax costatum, Daniellia oliveri, Khaya senegalensis, Pterocarpus erinaceus, Cordia sinensis, Prosopis africana et Acacia sieberiana sont aussi épargnés pour leur intérêt dans l'alimentation des bovins et sont le plus souvent rencontrées dans les champs des éleveurs. Afzelia africana est plus épargné dans les champs des éleveurs que ceux des agriculteurs et dans les jeunes jachères $(31,58 \%$ contre 10,52 et $9,6 \%$ respectivement).

La richesse spécifique est similaire (7 contre 8 et 9 espèces respectivement) dans les champs des éleveurs, des agriculteurs et dans les jeunes jachères alors qu'elle est de 4 espèces dans les vielles jachères. La densité des ligneux est de 38 pieds/ha dans les champs des agriculteurs et des éleveurs contre 31 et 34 pieds/ha dans les jeunes et vieilles jachères.

Les indices de diversité de Shannon $(\mathrm{H})$ et l'équitabilité de Pielou (E) calculés sont résumés dans le tableau 2.

La répartition par classe de diamètre des individus des divers ligneux épargnés est ajustée par une fonction polynomiale et a une courbe en forme de " cloche" (Figures 3 à 6), dans tous les terroirs agricoles étudiés. Les coefficients de détermination calculés pour chaque fonction polynomiale sont de 0,63 ; 0,$74 ; 0,74$ et 0,95 respectivement pour les vieilles jachères, les jeunes jachères, les champs des agriculteurs et les champs des éleveurs. 
Tableau 1 : Proportion (\%) des ligneux épargnés par espèce et par type de terroirs agricoles inventoriés.

\begin{tabular}{lcccc}
\hline Type de terroirs agricoles & $\begin{array}{c}\text { Champ des } \\
\text { agriculteurs }\end{array}$ & $\begin{array}{c}\text { Champ des } \\
\text { éleveurs }\end{array}$ & $\begin{array}{c}\text { Jeunes } \\
\text { jachères }\end{array}$ & $\begin{array}{c}\text { Vieilles } \\
\text { jachères }\end{array}$ \\
Espèces ligneuses & & & & \\
\hline Vitellaria paradoxa & 57,9 & 44,74 & 61,3 & 70,58 \\
Parkia biglobosa & 21,05 & 5,26 & 9,6 & 17,64 \\
Afzelia africana & 10,52 & 31,58 & 9,6 & - \\
Bombax costatum & 2,63 & 2,63 & - & 8,82 \\
Daniellia oliveri & - & 10,52 & - & - \\
Prosopis africana & - & 2,63 & 6,45 & - \\
Pterocarpus erinaceus & - & 2,64 & - & - \\
Autres* & * Les espèces présentes dans les placeaux inventoriés et dont la proportion est inférieure à 1\%. & \\
\hline \multicolumn{4}{c}{}
\end{tabular}

Tableau 2: Indice de Shannon et Equitabilité de Piélou par type de terroirs agricole.

\begin{tabular}{lcc}
\hline \multicolumn{1}{c}{ Indices de diversités } & $\begin{array}{c}\text { Indice de Shannon (H) } \\
\text { en bits }\end{array}$ & Equitabilité de Piélou (E) \\
Types de terroirs agricoles & 1,96 & 0,65 \\
\hline Champs des agriculteurs & 2,02 & 0,72 \\
Champs des éleveurs & 1,98 & 0,66 \\
Jeunes jachères & 1,24 & 0,62 \\
Vielles jachères & \\
\hline
\end{tabular}

\section{DISCUSSION}

\section{Analyse du système d'élevage}

En Afrique subsaharienne, la population rurale vit essentiellement de l'agriculture et de l'élevage. Ainsi les systèmes d'élevage qui existent combinent pour la plupart l'agriculture et l'élevage et sont diversifiés. Cirad (2006) distingue quatre (4) grands systèmes d'élevage de bovin: le système pastoral transhumant, le système agro-pastoral en zone d'agriculture-élevage, les systèmes semi-intensifs et les systèmes intensifs. Dans cette zone le système le plus observé est l'agro-pastoralisme pratiqué par les sédentaires (89\%). La valeur de la taille du cheptel obtenue est bien en relation avec un tel système et mode de conduite et corrobore la valeur trouvée par Djodjouwin (2001) qui est de 49 bovins par troupeau chez les sédentaires dans les forêts classées des MontsKouffé et Wari-Maro au Bénin. Ce système d'élevage en opposition au système pastoral transhumant, n'est pratiqué que sur des espaces pastoraux réduits et par ricochet les éleveurs ne peuvent qu'entretenir des petits effectifs. En effet selon Shazali et Abdel
Ghaffar (1999), l'une des causes de la transhumance est le problème de conservation d'un nombre maximum d'animaux pendant la saison sèche. Ainsi, les éleveurs autour des aires protégées du fait de la contrainte spatiale, n'entretiennent pas des effectifs importants. Ce comportement permet d'éviter la transhumance à travers l'aire protégée.

La pratique de ce système d'élevage dans la zone d'étude s'explique aussi par la place qu'occupe la production cotonnière. En effet la traction animale est ici bien intégrée dans le système de production culturale. Ainsi il existe une forte collaboration entre agriculteur et éleveur. Les agriculteurs achètent généralement leurs animaux de trait auprès des éleveurs ou bien leur confient aussi des bovins pour l'élevage excepté les animaux de trait qu'ils gardent eux-mêmes. Quant aux éleveurs, ils bénéficient de ce système, en écoulant certains de leurs animaux par des relations de proximité avec des agriculteurs. Aussi les éleveurs utilisent-ils les sous produits de récolte de leurs champs et des champs des agriculteurs. 


\section{T. D. HOUEHANOU et al. / Int. J. Biol. Chem. Sci. 2(4): 497-507, 2008}

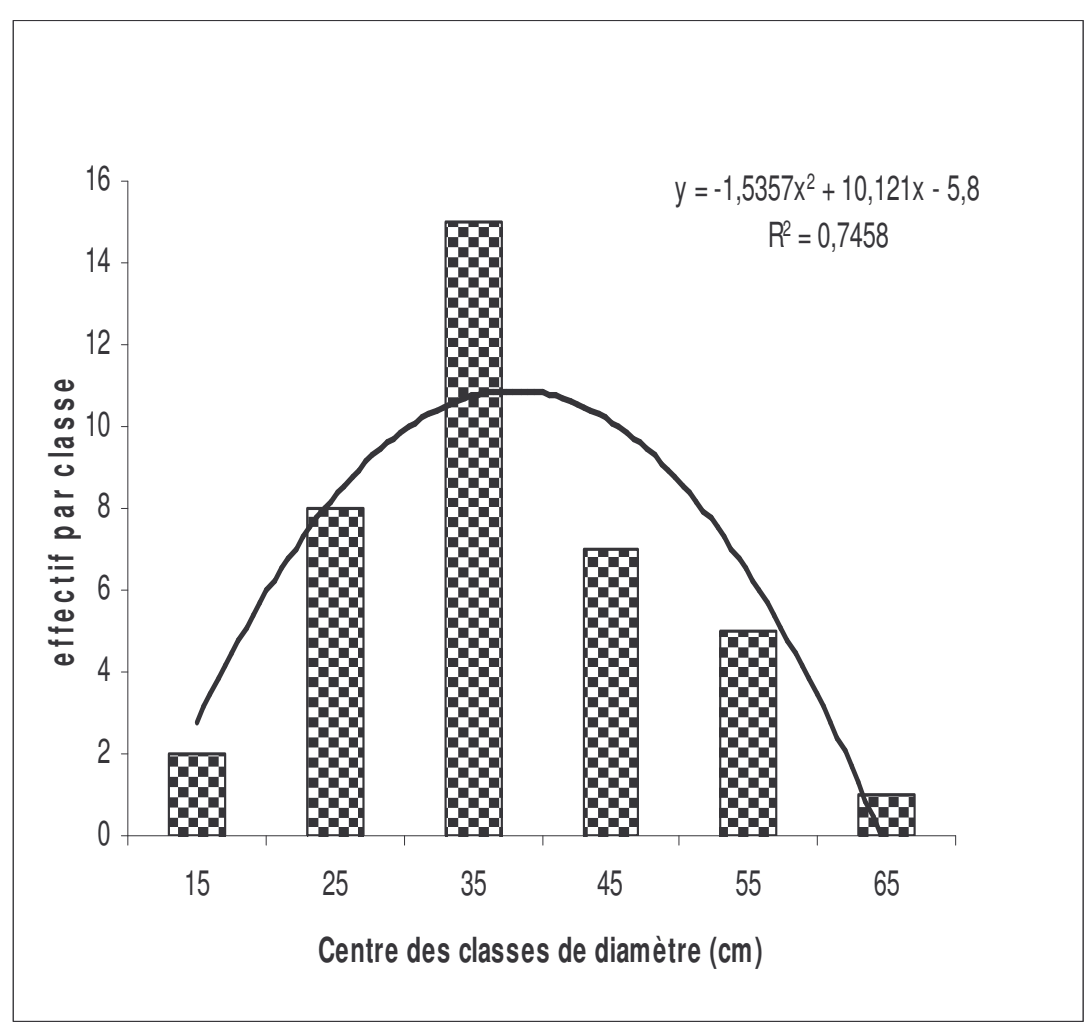

Figure 3 : Répartition des individus par classe de diamètre dans les champs des agriculteurs.

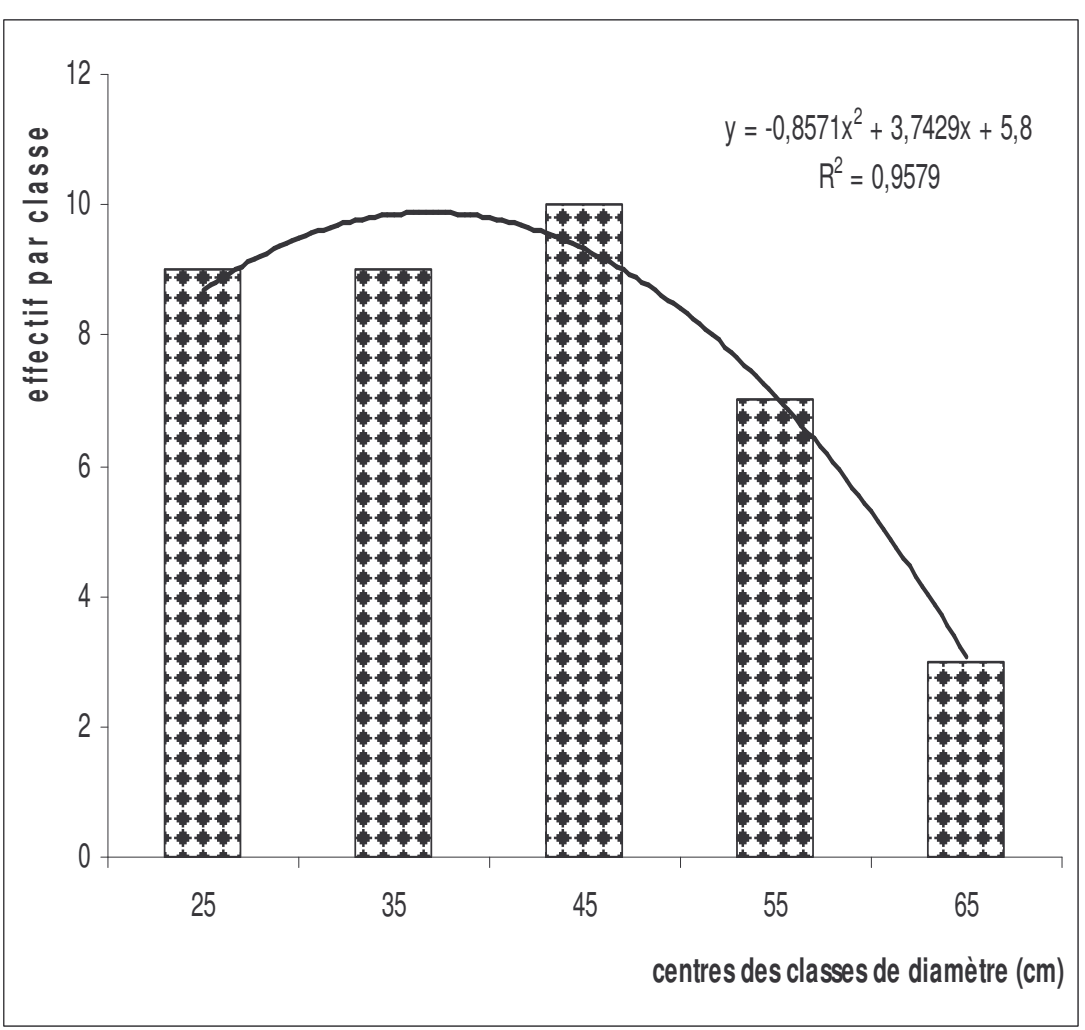

Figure 4 : Répartition des individus par classe de diamètre dans les champs des éleveurs. 
T. D. HOUEHANOU et al. / Int. J. Biol. Chem. Sci. 2(4): 497-507, 2008

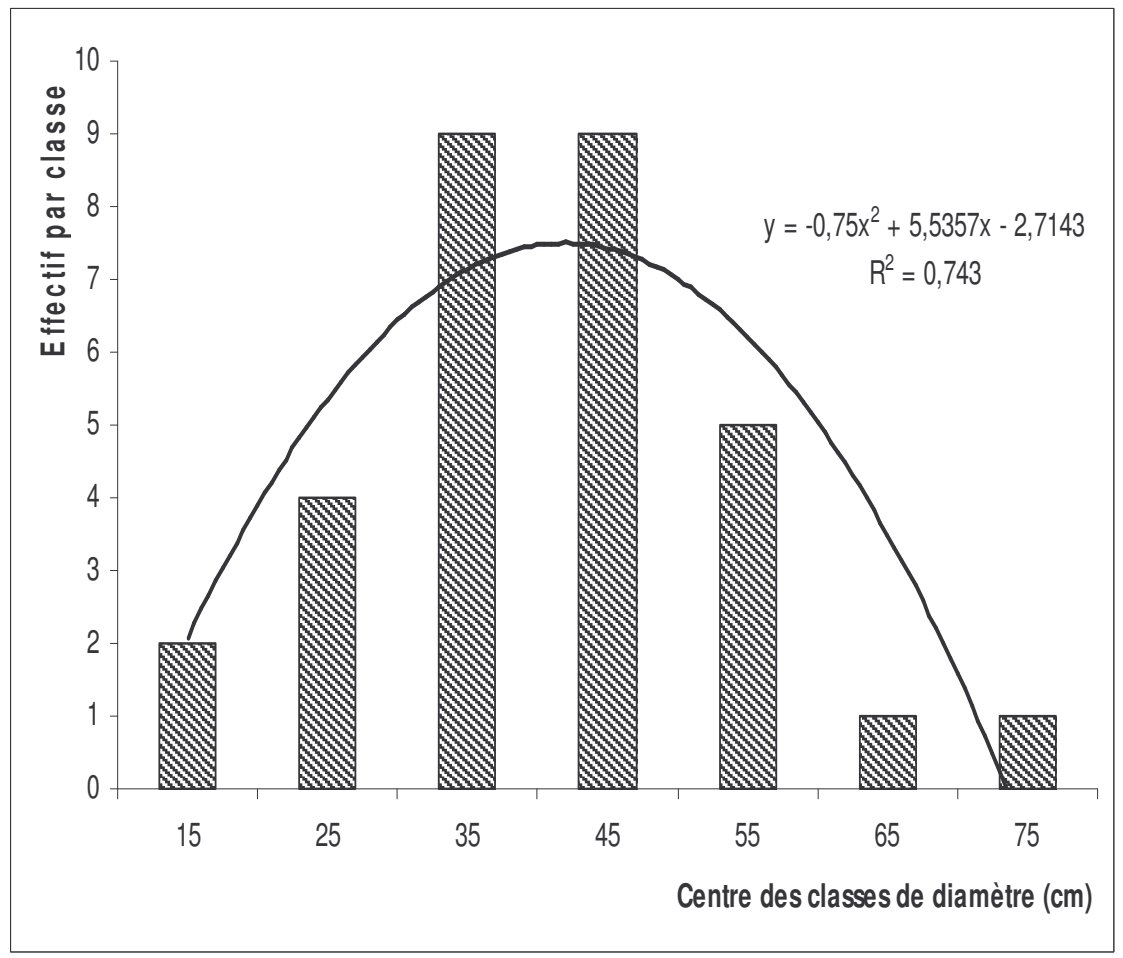

Figure 5 : Répartition des individus par classe de diamètre dans les jeunes jachères.

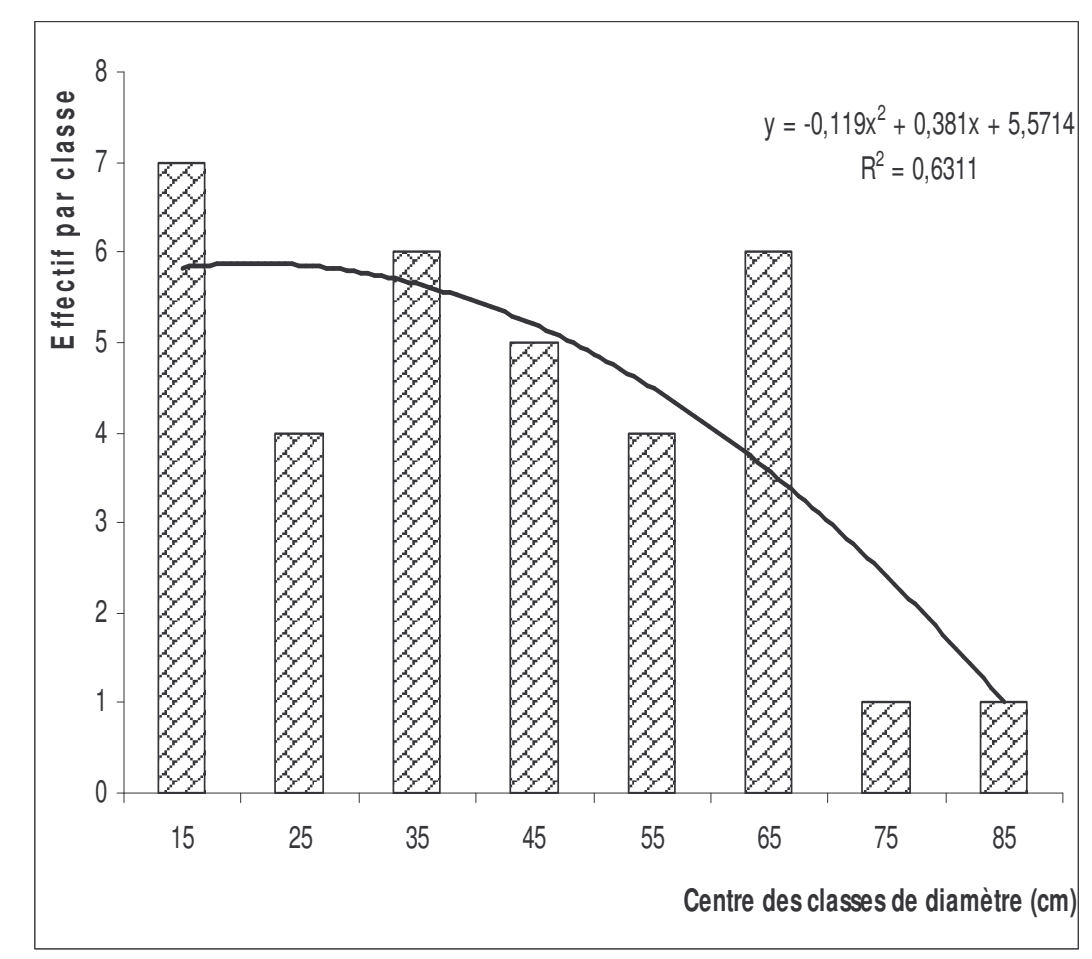

Figure 6 : Répartition des individus par classe de diamètre dans les vieilles jachères. 
En ce qui concerne la transhumance pratiquée par seulement $11 \%$ des éleveurs enquêtés, il faut noter que ceux-ci descendent jusqu'à la partie centrale du pays à la recherche de pâturage en période de déficit et ne traversent guère la Réserve du W. Ceci est contraire au phénomène observé dans la partie périphérique Nord (Karimama et Mallanville) de la Réserve. En effet, Sounkéré (2003) a montré que c'est dans cette partie périphérique Nord du $\mathrm{W}$ que se trouvent les couloirs d'entrée et itinéraires de transhumance. Aussi signale-t-il que ces transhumants sont plus des étrangers.

\section{Analyse de la structure des terroirs agricoles inventoriés}

Le karité et le néré sont les ligneux plus épargnés pour leur importance socioéconomique. Cet intérêt s'explique d'une part, par l'amélioration du revenu des femmes qui commercialisent les noix de karité ou les transforment en beurre de karité principale source de lipide dans la zone et d'autre part, par la fabrication du "Afitin" (moutarde locale) à partir des graines de néré. De ces deux espèces ligneuses, le karité (Vitellaria paradoxa) a une forte représentation dans tous les terroirs agricoles inventoriés. En effet, sa productivité en fruit est améliorée dans les parcs agro-forestiers par rapport aux formations naturelles (Lamien et al., 2004) et chaque groupe socio-professionnel trouve un intérêt à l'épargner d'une manière ou d'une autre afin d'augmenter sa production annuelle.

Les richesses spécifiques obtenues (7 contre 8 et 9 espèces respectivement) dans les champs des éleveurs, des agriculteurs et dans les jeunes jachères corroborent les résultats de Wala et al. (2005) qui trouvent des valeurs de $9,7,5$ et 8 espèces respectivement dans les parcs à Parkia biglobosa; dans les parcs mixtes à Parkia biglobosa et Adansonia digitata; dans les parcs mixtes à Parkia biglobosa et Vitellaria paradoxa et dans les parcs mixtes composites au Nord du Togo. Dans les vieilles jachères la richesse spécifique a été de 4 espèces et serait, due à la non prise en compte de la régénération potentielle. Dans les jachères en général et les vieilles en particulier, cette régénération est plus importante.

Les valeurs de densité obtenues (38, 31 et 34 pieds/ha) sont similaires à celle de Wala et al. (2005) dans les parcs mixtes composites (32 pieds/ha) au Nord du Togo, comparables à la valeur minimale de Agbahungba et Depommier (1999) dans la région du Borgou au Bénin (40 arbres/ha). De même les valeurs des indices de diversité trouvées sont similaires à celles de Wala et al. (2005) qui trouvent 1,$7 ; 1,2 ; 1,6$ et 2,2 bits respectivement dans les parcs à Parkia biglobosa, dans les parcs mixtes à Parkia biglobosa et Vitellaria paradoxa, dans les parcs mixtes à Parkia biglobosa et Adansonia digitata puis dans les parcs mixtes composites. Les valeurs d'indice les plus élevées $(H=2,02$ bits et $E=0,72)$ obtenues dans les champs des éleveurs font de ce terroir agricole celui le plus diversifié. Les éleveurs épargnent les ligneux tels que Afzelia africana, Pterocarpus erinaceus, Daniellia oliveri, Bombax costatum, qui ont une importance dans l'alimentation de leurs animaux. Ces ligneux ajoutés aux Vitellaria paradoxa et Parkia bigbobosa, rendent leur champ plus diversifié comparativement à celui des agriculteurs. Afzelia africana en particulier est plus épargné dans les campements et dans les champs des éleveurs que les autres ligneux fourragers. Ceci peut s'expliquer par son importance pour la production du lait (selon les éleveurs enquêtés) qui est d'une grande importance socio-économique dans les ménages peuls.

\section{Conclusion}

La gestion de l'espace pastoral autour de la zone cynégétique de la Djona montre la dominance du sédentarisme pratiqué par des agro-pasteurs qui peuvent être soit des Peuls, des Monkolés ou des Dendis. Quant à la structure des terroirs agricoles, les peuls conservent plus les ligneux fourragers dans leur champ contrairement aux Monkolés et Dendis. Ceci implique une densité plus grande de ces ligneux dans les champs des éleveurs que dans les champs des agriculteurs. De ces ligneux, Afzelia africana est celui le plus recherché dans ces systèmes d'élevage bovin. Selon les éleveurs enquêtés, ce ligneux favoriserait la production laitière.

Aussi cette étude a-t-elle montrée que la rareté des ligneux fourragers les plus prisés (Afzelia africana, Khaya senegalensis et Pterocarpus erinaceus) pousse déjà les éleveurs à l'émondage d'autres ligneux tels 
que, Bombax costatum, Daniellia oliveri, Cordia sinensis, Prosopis africana et Acacia sieberiana.

\section{REMERCIEMENTS}

Nos gratitudes vont à l'endroit du département de Production Animale de la Faculté des Sciences Agronomiques (FSA) de l'Université d'Abomey Calavi (UAC) pour son assistance technique; de toutes les personnes qui nous ont aidés sur le terrain pour la collecte des informations; des peuls qui nous sont restés hospitaliers et de l'institution ANAFE (African Network for Agroforestry Education) pour son soutien financier.

\section{REFERENCES BIBLIOGRAPHIQUES}

Adandédjan CC. 1999. L'agroforesterie pour une Utilisation et une Gestion Durable des Terres et des Ressources Naturelles en Afrique Sub-Saharienne Humide. ICRAF.DSO : Kumassi, Ghana ; 8p.

Adjakpa JB. 1989. Etude écologique de la zone cynégétique de la Djona (Province de Borgou). Rapport sur une mission accomplie par la SEAPA/PAPM 60 p.

Agbahungba G, Depommier D. 1999. Aspects du parc à karités-nérés (Vitellaria paradoxa Gaertu F. Parkia bigbobosa Jacques ; Beuth) dans le sud du Borgou (Bénin). Bois For. Trop., 222: 41-54.

Brisso N, Houinato M, Adandédjan C, Sinsin B. 2007. Dry season woody fodder productivity in savannas. Short communication. Ghanaian Journal of Animal Science, 2 et 3 (1): 181-185.

Chaibou M. 2005. Productivité zootechnique du désert: le cas du bassin laitier d'AGADEZ au Niger. Thèse Doctorat ès science, Université de Montpellier II, $310 \mathrm{p}$.

Cirad. 2006. L'élevage Ouest-Africain : Déficit d'organisation. L'information Agricole n॰801: 28 - 29.

Djodjouwin L. 2001. Etude sur les aménagements écotouristiques et la gestion pastorale dans les terroirs et forêts classée des Monts-Kouffé de WariMaro. Mémoire de DESS, 77p.

Faye B. \& Alary V. 2001. Les enjeux des productions animales dans les pays du sud. INRA Prod. Anim., 14: 3-13. In www.inra.fr
Houinato M. 2001. Phytosociologie, écologie, production et capacité de charge des formations végétales pâturées dans la région des Monts Kouffé (Bénin) Th. Doc. Uni. Li. Br., 219 p.

Lamien N, Ouédraogo SJ, Diallo OB, Guinko S. 2004. Productivité fruitière du karité (Vitellaria paradoxa Gaertn. C. F, Sapotaceae) dans les parcs agroforestiers traditionnels au Burkina Faso. Fruits, 59: 423-429.

Le Houerou HN, 1980. Le rôle des ligneux dans les zones sahéliennes. In Le Houerou H.N. éd. Les Fourrages Ligneux en Afrique. Etat Actuel des Connaissances. Ilca: Addis Abeba, Ethiopie; 85-101.

MDR. 1994. Définition d'une stratégie et d'un plan d'action pour le secteur de l'élevage. Volume1, rapport principal ; MDR, Cotonou, 95p.

Ndzingu Awa D., Njoya A., Logtene M, Bechir Ali B., Delafosse Arnaud \& Maho A. 2004. Contraintes, opportunités et évolution des systèmes d'élevage en zone semi-aride des savanes d'Afrique centrale. Agricultures, 13(4): 331-340.

Piot J, 1980. Les méthodes de gestion et d'exploitation des fourrages ligneux : peuplements naturels et plantations artificielles. In les Fourrages Ligneux en Afrique: Etat Actuel des Connaissances. CIPEA; 335-343.

Sacko B. 1991. Caractérisation des Disponibilités Fourragères Ligneuses sur des Parcours Naturels Sahéliens Exploités par des Bovins, Ovins ou Caprins. ISRA-IERSERZ; 100 p.

Shazali S, Abdel Ghaffar MA. 1999. Régimes fonciers et pastoralismes dans les zones agricoles en expansion: Soudan et corne de l'Afrique. International Institute for environment and development. Programmes Zones Arides, Londres, 20p.

Sieglstetter R, Witting R. 2001. Utilisation des ligneux sauvages et son effet sur la végétation dans la région d'Atakora (Bénin Nord occidental). Etudes flor. Veg. Burkina Faso n ${ }^{\circ}$ 7: 23- 30.

Sinsin B. 1991. Exploitation des pâturages dans un système traditionnel d'élevage bovin dans le périmètre Nikki-Kalalé au Bénin. Acte de la IVème Conférence 
Internationale des Terres de Parcours. Montpellier, France, pp 660-662.

Sounkéré K. 2003. Analyse des formes d'utilisation de l'espace dans les terroirs agro-pastoraux de la zone périphérique du Parc National du W au Bénin : cas de la commune de Karimama. Mémoire d'Ing. Agr., Université d'Abomey-Calavi, p. 51. Wala K, Sinsin B, Guelly KA, Kokou K, Akpagana K. 2005. Typologie et structure des parcs agro-forestiers dans la préfecture de Doufelgou (Togo) Sécheresse, 16(3): 209-216. 